\title{
Association Between Pretreatment Serum Apolipoprotein A1 and Prognosis of Solid Tumors in Chinese Population: A Systematic Review and Meta-Analysis
}

\author{
Jiayuan Wu $u^{a, b} \quad$ Chuanmeng Zhang ${ }^{c}$ Gaohua Zhang ${ }^{d} \quad$ Yufeng Wang ${ }^{e}$ \\ Zhanhui Zhang ${ }^{\text {e Wenmei Su }}{ }^{f}$ Jun Lyu
}

\begin{abstract}
${ }^{a}$ Clinical Research Center, The First Affiliated Hospital of Xi'an Jiaotong University, Xi'an, 'Department of Clinical Research, The Affiliated Hospital of Guangdong Medical University, Zhanjiang, 'Department of Central Laboratory, People's Hospital of Taizhou, Taizhou, 'Hospital Office, People's Hospital of Gaozhou, Gaozhou, eSchool of Public Health, Guangdong Medical University, Zhanjiang, 'Department of Oncology, The Affiliated Hospital of Guangdong Medical University, Zhanjiang, China
\end{abstract}

\section{Key Words}

Apolipoprotein A1 • Prognosis • Solid tumor • Chinese population • Meta-analysis

\begin{abstract}
Background/Aims: Serum apolipoprotein A1 (apoA1) has been reported to be abnormally expressed in several malignancies. However, the prognostic role of apoA1 in solid tumors is still controversial. We conducted this meta-analysis to obtain a more accurate evaluation of prognostic significance of apoA1 in Chinese patients with solid tumors. Methods: A comprehensive literature search of electronic databases was carried out up to August 2018. We included studies investigating the association between pretreatment serum apoA1 level and clinicopathological features, including survival outcomes, in solid tumors. Hazard ratios (HRs) and odds ratio (ORs) with 95\% confidence intervals (Cls) were applied as effect size estimates. Results: A total of 13 studies and 8052 patients were included in our meta-analysis. Elevated level of pretreatment serum apoA1 was markedly associated with an improved OS (pooled $\mathrm{HR}=0.608,95 \% \mathrm{Cl}=0.557-0.665, P<0.001)$. The statistical significances were observed in all cancer types, including digestive system malignancies (pooled $\mathrm{HR}=0.633 ; 95 \% \mathrm{Cl}=$ $0.550-0.727 ; P<0.001$ ), urinary system cancers (pooled $\mathrm{HR}=0.471 ; 95 \% \mathrm{Cl}=0.352-0.630 ; P<$ 0.001 ), nasopharyngeal cancer (pooled $\mathrm{HR}=0.642 ; 95 \% \mathrm{Cl}=0.538-0.766 ; P<0.001$ ) and nonsmall cell lung cancer (pooled $\mathrm{HR}=0.526 ; 95 \% \mathrm{Cl}=0.329-0.841 ; P=0.007$ ), but not in breast cancer (pooled HR $=0.573 ; 95 \% \mathrm{Cl}=0.266-1.246 ; P=0.155$ ). Meanwhile, cancer patients with a low level of serum apoA1 suffered an unfavorable DFS (pooled $\mathrm{HR}=0.714,95 \% \mathrm{Cl}=0.603-$ $0.845, \mathrm{P}<0.001)$. Moreover, abnormal serum apoA1 was significantly correlated to tumor size (pooled $\mathrm{OR}=0.640,95 \% \mathrm{Cl}=0.475-0.863, P=0.003$ ), tumor differentiation (pooled HR = J. Wu, C. Zhang, and G. Zhang contributed equally to this work.

Jun Lyu Clinical Research Center, The First Affiliated Hospital of Xi'an Jiaotong University

and Wenmei Su

Xi'an 710061, Shaanxi (China)

Tel.+86-029-85323614, E-Mail lujun2006@xjtu.edu.cn; suwenmei123@hotmail.com
\end{abstract}




\section{Cellular Physiology Cell Physiol Biochem 2018;51:575-588 and Biochemistry Published \begin{tabular}{l|l} 
DOI: 10.1159/000495277 & $\begin{array}{l}\text { (c) } 2018 \text { The Author(s). Published by S. Karger AG, Basel } \\
\text { www.karger.com/cpb }\end{array}$
\end{tabular} \\ Wu et al.: Prognostic Role of Serum Apolipoprotein A1 in Solid Tumors}

$0.724,95 \% \mathrm{Cl}=0.565-0.929, P=0.011$ ), and tumor stage (pooled $\mathrm{HR}=0.493,95 \% \mathrm{Cl}=0.384$ $-0.633, P<0.001$ ). Conclusion: Elevated level of pretreatment serum apoA1 was significantly associated with longer survival in patients with solid tumors. Pretreatment serum apoA1 could serve as a novel positive factor for malignant patient prognosis in Chinese population.

(C) 2018 The Author(s)

Published by S. Karger AG, Basel

\section{Introduction}

Metabolic reprogramming is one of the crucial hallmarks that distinguish cancer cells from normal cells and rewired to the demands of cell proliferation and survival [1]. This metabolic property of cancer cells not only meets the growth requirement of energy supplement but also supports the increased need of metabolic intermediates for the biosynthesis of cellular building blocks and signaling molecules [2]. A shift towards aerobic glycolysis with high glucose uptake even under sufficient oxygen is one of the most prominent characteristic of metabolic alterations in cancer. This phenomenon is called the "Warburg effect" [3]. Glucose was once considered as the major fuel, if not the only factor, that support tumor growth [4]. Malignant cells are now known to employ a wide variety of substrates to support their multiplication [5].

Lipids, also known as fats, are energy-rich molecules that serve as important energy sources for cellular metabolic processes when nutrients are limited. Lipids also function as structural components of all membranes and second messengers of cell signaling. Lipids in mammalian cells are mainly derived from de novo synthesis and food intake. Abnormality in lipid metabolism contributes to various metabolic diseases, such as cardiovascular diseases, obesity, hepatic steatosis, and diabetes [6-9]. Lipid metabolism is significantly enhanced during carcinogenesis, because most lipogenic enzymes are upregulated or activated in cancer cells [10]. Furthermore, lipids also promote cancer development, invasion, and metastasis, because highly proliferative malignant cells usually exhibit strong lipid avidity by increasing either the endogenous synthesis or the exogenous uptake of lipids [11]. Thus, targeting lipid metabolism reprogramming might be a promising strategy for anticancer therapy.

Extensive studies have shown that an abnormal level of lipid profile may influence carcinogenesis and is closely linked to tumor progression [12]. Cholesterol synthesis is raised in cancer cells compared with normal cells, as tumor cells require excess cholesterol and intermediates of the cholesterol biosynthesis pathway to sustain a high level of proliferation [13]. Cholesterol from non-hepatic peripheral tissues is transported through the hepatobiliary tract to the intestinal lumen. This process leads to fecal excretion, which is known as reverse cholesterol transport (RCT) mediated by high-density lipoprotein (HDL) at its initial step. As a major protein component of HDL, apolipoprotein A1 (apoA1) constitutes approximately $70 \%$ of the protein content of HDL particles, defining its size and shape, solubilizing its lipid component, and helping in RCT [14]. ApoA1 also functions as a cofactor of lecithin cholesterol acyltransferase (LACT) and participates in the formation of most plasma cholesteryl ester [15]. Accumulating evidence has revealed that serum apoA1 is inversely correlated with the risk of developing breast, colorectal, lung, and ovarian cancer [16-18]. Elevated apoA1 transgenic expression has shown a reduction of tumor burden and a survival benefit in mouse models of ovarian cancer and melanoma $[19,20]$. The US Food and Drug Administration also approved the application of apoA1 along with transthyretin, transferring, $\beta 2$-microglobulin, prealbumin, and CA125 for detecting early stage ovarian cancer, and these combined biomarkers test is known as OVA ${ }^{\circledR}[21,22]$. Several publications have recently reported that low serum apoA1 level was associated with poor prognosis of solid tumors in Chinese population. However, several studies claimed that the connection was nonsignificant. Thus, the prognostic role of apoA1 in solid tumors has not yet been fully elucidated. Evidence from individual study is insufficient to conclude whether apoA1 can be 


\section{Cellular Physiology Cell Physiol Biochem 2018;51:575-588

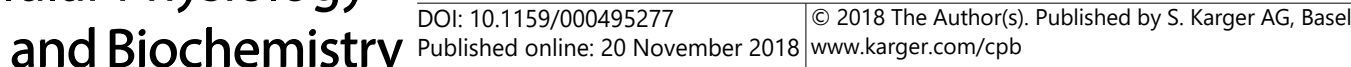

Wu et al.: Prognostic Role of Serum Apolipoprotein A1 in Solid Tumors

used as an effective predictive factor of prognosis. Hence, we conducted this meta-analysis to derive a more precise estimation of the role of apoA1 in the survival outcomes of patients with solid malignances.

\section{Materials and Methods}

\section{Search strategy}

This meta-analysis was strictly conducted according to the Preferred Reporting Items for Systematic Review and Meta-Analyses (PRISMA) guidelines [23]. A comprehensive literature searching was performed in several computerized databases, such as PubMed, Embase, Web of Science, Science Citation Index, and Cochrane Library, as well as three Chinese databases, including China National Knowledge Internet (CNKI), Wanfang and Weipu databases. The key terms were used as follow: ("apolipoprotein A1" or "apoA1" or "apolipoprotein A-I" or "apo A-I") and ("cancer" or "tumor" or "carcinoma" or "neoplasm") and ("prognosis" or "survival" or "outcome"). The last search date was $1^{\text {st }}$ Aug 2018. The references of the retrieved articles were also manually examined in order to discover additional relevant publications.

\section{Study selection}

Two researchers (WJY and ZCM) independently carried out the study selection, and all disagreements were resolved by discussion. Eligible studies for this meta-analysis had to meet the following criteria: 1) cohort studies investigated the association between pretreatment serum apoA1 levels and survival outcomes of solid tumors, such as overall survival (OS) and disease free survival (DFS);2) studies originated from China and focused on Chinese population; 3) patients were divided into high apoA1 group and low (normal) apoA1 group, regardless of the cutoff value; 4) hazard ratios (HRs) and the corresponding 95\% confidence intervals (CIs) could be extracted or estimated from the original data; and 5) publications were written in any language as full papers. Articles were excluded if they were: 1) case reports, reviews, conference abstracts, or nonoriginal articles; 2) articles of patients with hematological malignancies; 3) duplicate publications; and 4) basic research or animal experiments.

\section{Data extraction and quality assessment}

Two researchers (WYF and ZCM) independently extracted data from all enrolled studies, and any disagreement was resolved by a third person (WJY). The following data was collected: first authors, year of publication, study region, duration time, cancer type, follow-up period, primary treatment, sample size, cutoff value, analysis method, clinicopathological features, survival outcome, HR and the corresponding $95 \%$ $\mathrm{CI}$, and quality score. In studies where the HR estimations were both provided in univariate and multivariate analyses, only the latter was applied to the data synthesis.

The quality of included studies was assessed by the Newcastle-Ottawa Scale (NOS) for cohort studies. The total score of NOS ranged from 0 to 9 , and studies with at least seven scores were considered to be of high quality.

\section{Statistical analysis}

STATA 12.0 software (STATA Corp., College Station, TX) was adopted to perform the statistical analyses. Pooled HRs and their 95\% CIs were applied to describe the association between apoA1 level and prognosis. HR values $>1$ suggested an increased risk of poor survival, while HR values $<1$ implied a survival benefit in patients with elevated serum apoA1 level. The result was considered statistically significant if the $95 \% \mathrm{CI}$ did not overlap 1 . Heterogeneity was qualitatively examined through the chi-squared $Q$ test and quantitatively estimated using the $I^{2}$ metric $\left(I^{2}<25 \%\right.$, no heterogeneity; $I^{2}=25 \%-50 \%$, moderate heterogeneity; $I^{2}>$ $50 \%$, extreme heterogeneity) [24]. When significant heterogeneity was observed $\left(P<0.05\right.$ or $\left.I^{2}>50 \%\right)$, a random-effects model was used to calculate the pooled HR estimation. Otherwise, a fixed-effects model was applied. Subgroup analyses and meta-regression analysis were performed to investigate the suspected factors for heterogeneity. Sensitivity analysis was conducted to evaluate the stability of the outcomes. Potential publication bias was quantitatively evaluated through Begg's and Egger's asymmetry tests [25], and was visually evaluated by funnel plots. If significant publication bias existed, trim and fill method was performed to validate the robustness of the meta-analysis results [26]. 


\section{Results}

\section{Study characteristics}

A total of 843 studies were identified from the initial electrical literature search, and then 732 papers were removed because of obvious irrelevance. After carefully screening the titles and abstracts, 78 papers were further excluded according to the inclusion and exclusion criteria. For the remaining potential candidate studies, 20 publications were removed after full text evaluation as follow: 11 articles that did not perform survival analysis; 5 papers that did not have sufficient data for HR calculation; 3 reports focused on other survival outcomes; and 1 study that originated from Caucasian population. Thus, 13 publications published from 2014 to 2018 were eligible for our meta-analysis [27-39]. The selection process is shown in Fig. 1.

The baseline characteristics of the included studies are summarized in Table 1 . The included studies originated from Guangdong [27, 29-36, 39], Beijing [28], and Shanghai [37, 38]. A total of 8052 patients diagnosed with several kinds of cancers were including. These cancers included gastric cancer (GC) [27], bladder cancer [28], non small cell lung cancer (NSCLC) [29,38], nasopharyngeal carcinoma (NPC) [30,39], breast cancer [31,32], colorectal cancer (CRC) [33], upper tract urothelial carcinoma (UTUC) [34], esophageal squamous cell cancer (ESCC) [35], renal cell carcinoma (RCC) [36], and hepatocellular carcinoma (HCC) [37]. Eleven studies were published in English [27-33, 35-37, 39], whereas the other two studies were in Chinese [34, 39]. One of these studies contained two cohorts [37]. Therefore, 14 cohorts were enrolled, and the sample sizes ranged from 99 to 1927. The outcomes of OS were reported in all 14 cohorts, and the end points of DFS were presented in 4 cohorts [30-32, 36]. Nine cohorts recruited patients with all disease stages (stage I - IV) [27, $29,30,32,34-37]$, three cohorts included patients with advanced stage (including stage IV [33, 39], and stage IIB - IV [38]), one cohort contained patients with stage I - III [31], and the other one reported the data of patient with stage Tis - T1 [28]. The median follow-up time ranged from 20.3 months to 89 months, and even five studies did not report it. The primary treatments extremely varied among the 14 cohorts. These treatments included surgery [27, $28,31,32,34-37]$, chemotherapy $[33,39]$, radiotherapy [30], and mixed treatment $[29,38]$. The HRs and their 95\% CIs in 11 cohorts were directly extracted from multivariate analyses, while those of the other cohorts were obtained from univariate analyses [27, 29, 31]. All cohort studies were of high quality and had seven scores or more in the quality criteria.

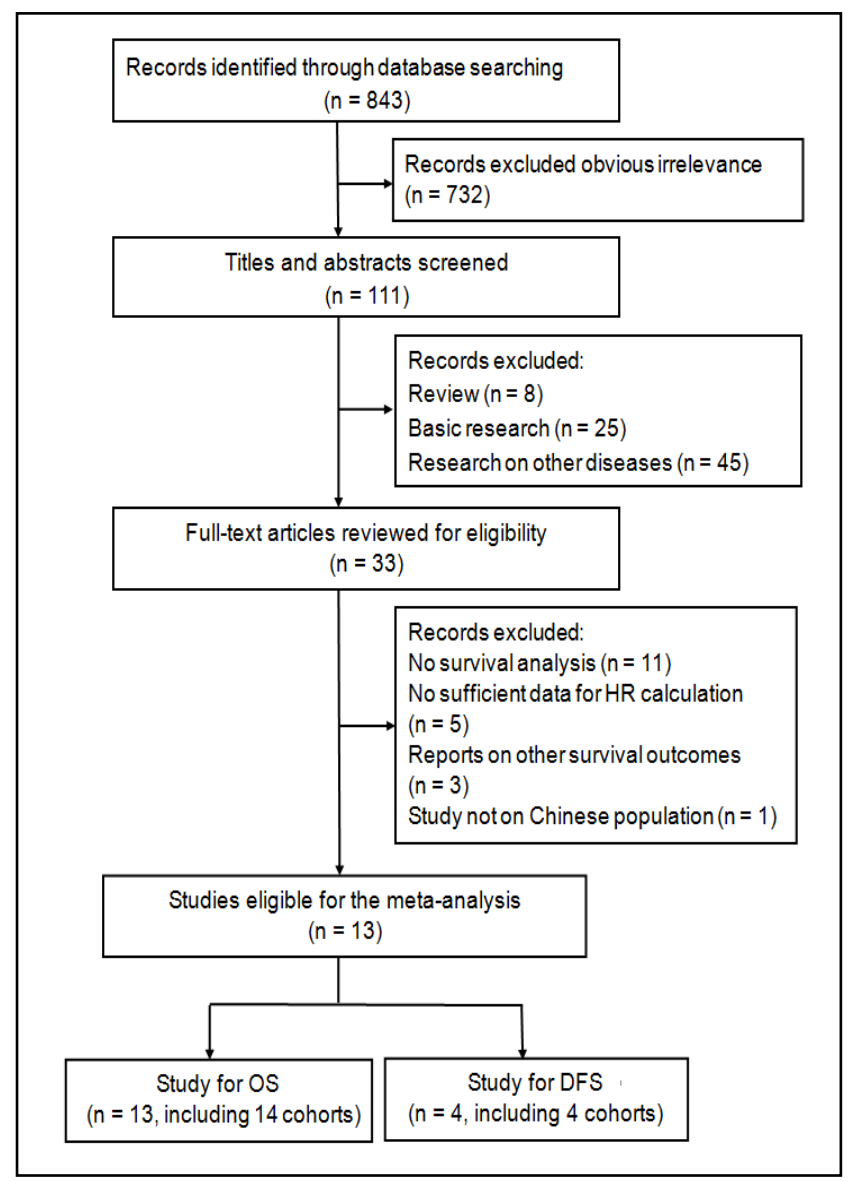

Fig. 1. Flow diagram of the study selection process. Abbreviation: HR, hazard ratio; OS, overall survival; DFS, disease free survival. 


\section{Cellular Physiology Cell Physiol Biochem 2018;51:575-588 and Biochemistry \begin{tabular}{l|l} 
DOI: $10.1159 / 000495277$ & $\begin{array}{l}\text { C } 2018 \text { The Author(s). Published by S. Karger AG, Basel } \\
\text { www.karger.com/cpb }\end{array}$
\end{tabular} \\ Wu et al.: Prognostic Role of Serum Apolipoprotein A1 in Solid Tumors}

Table 1. Main characteristic of the eligible studies. ${ }^{\text {a }}$ First of two cohorts in this study; ${ }^{\mathrm{b}}$ second of two cohorts in this study.Abbreviations: NR, none reported; GC, gastric cancer; NSCLC, non small cell lung cancer; NPC, nasopharyngeal carcinoma; CRC, colorectal cancer; UCUC, Upper tract urothelial carcinoma; ESCC, esophageal squamous cell cancer; RCC, renal cell cancer; HCC, hepatocellular carcinoma; OS, overall survival; DFS, disease free survival

\begin{tabular}{|c|c|c|c|c|c|c|c|c|c|c|c|c|}
\hline $\begin{array}{l}\begin{array}{l}\text { Author } \\
\text { (year) }\end{array} \\
\end{array}$ & $\begin{array}{c}\text { Study } \\
\text { region }\end{array}$ & Duration & $\begin{array}{c}\text { Cancer } \\
\text { type }\end{array}$ & $\begin{array}{c}\text { Clinical } \\
\text { stage }\end{array}$ & $\begin{array}{c}\text { Primary } \\
\text { treatment }\end{array}$ & $\begin{array}{c}\begin{array}{c}\text { Follow up } \\
\text { (month) }\end{array} \\
\end{array}$ & $\begin{array}{c}\text { Sample } \\
\text { size }\end{array}$ & $\begin{array}{l}\text { Cutoff, g/L } \\
\text { (high/low) }\end{array}$ & $\begin{array}{c}\text { Analysis } \\
\text { method }\end{array}$ & Outcome & Language & Quality \\
\hline $\begin{array}{l}\text { Ma MZ } \\
\text { (2018) [27] }\end{array}$ & Guangdong & $2005 \sim 2010$ & GC & I $\sim$ IV & Surgery & $\begin{array}{l}\text { Up to Mar } \\
2017\end{array}$ & 1201 & $1.4(246 / 955)$ & Univariate & os & English & 7 \\
\hline $\begin{array}{l}\text { Shang ZH } \\
\text { (2018) [28] }\end{array}$ & Beijing & $2001 \sim 2011$ & $\begin{array}{l}\text { Bladder } \\
\text { cancer }\end{array}$ & Tis $\sim$ T1 & Surgery & $\begin{array}{c}\text { Median } 89 \\
(10-154)\end{array}$ & 470 & $\begin{array}{c}1.19 \\
(114 / 326)\end{array}$ & Multivariate & os & English & 9 \\
\hline $\begin{array}{l}\text { Chen SL } \\
\text { (2018) [29] }\end{array}$ & Guangdong & $2008 \sim 2010$ & NSCLC & I $\sim$ IV & Mixed & Median 33 & 141 & $1.17(85 / 56)$ & Univariate & os & English & 7 \\
\hline $\begin{array}{l}\text { Chang H } \\
\text { (2018) [30] }\end{array}$ & Guangdong & $2010 \sim 2011$ & NPC & I $\sim$ IV & Radiotherapy & $\begin{array}{c}\text { Median } 66.2 \\
(5.97-89.7)\end{array}$ & 1927 & $\begin{array}{c}1.125 \\
(1160 / 767)\end{array}$ & Multivariate & OS, DFS & English & 8 \\
\hline $\begin{array}{l}\mathrm{Li} \mathrm{X} \mathrm{(2017)} \\
\text { [31] }\end{array}$ & Guangdong & $2008 \sim 2011$ & $\begin{array}{l}\text { Breast } \\
\text { cancer }\end{array}$ & I $\sim$ III & Surgery & $\mathrm{NR}$ & 1044 & $\begin{array}{c}1.56 \\
(197 / 847)\end{array}$ & Univariate & OS, DFS & English & 8 \\
\hline $\begin{array}{l}\text { Lin XR } \\
\text { (2017) [32] }\end{array}$ & Guangdong & $2007-2011$ & $\begin{array}{l}\text { Breast } \\
\text { cancer }\end{array}$ & I $\sim$ IV & Surgery & Median 78 & 299 & $1.12(244 / 55)$ & Multivariate & OS, DFS & English & 7 \\
\hline $\begin{array}{l}\text { Quan Q } \\
\text { (2017) [33] }\end{array}$ & Guangdong & $2005 \sim 2013$ & CRC & IV & Chemotherapy & $\begin{array}{c}\text { Median } 46(1- \\
131)\end{array}$ & 508 & $\begin{array}{c}1.105 \\
(254 / 254)\end{array}$ & Multivariate & os & English & 8 \\
\hline $\begin{array}{l}\text { Wen WJ } \\
\text { (2016) [34] }\end{array}$ & Guangdong & $2000 \sim 2012$ & UTUC & I $\sim$ IV & Surgery & $\begin{array}{c}\text { Median } 51.5 \\
(11-159)\end{array}$ & 99 & $1.06(61 / 38)$ & Multivariate & os & Chinese & 8 \\
\hline $\begin{array}{l}\text { Wang XP } \\
\text { (2016) [35] }\end{array}$ & Guangdong & $2007 \sim 2009$ & ESCC & I $\sim$ IV & Surgery & $\begin{array}{l}\text { Up to Oct } \\
2015\end{array}$ & 210 & $\begin{array}{c}1.21 \\
(112 / 108)\end{array}$ & Multivariate & os & English & 7 \\
\hline $\begin{array}{l}\text { Guo SJ } \\
\text { (2016) [36] }\end{array}$ & Guangdong & $2000 \sim 2012$ & RCC & I $\sim$ IV & Surgery & $\begin{array}{l}\text { Median 76.2 } \\
(50.0-107.5)\end{array}$ & 786 & $\begin{array}{c}1.04 \\
(579 / 207)\end{array}$ & Multivariate & OS, DFS & English & 7 \\
\hline $\begin{array}{l}\mathrm{Ma} \mathrm{XL} \\
(2016)^{\text {a }} \\
{[37]}\end{array}$ & Shanghai & 2012 & HCC & $\mathrm{I} \sim \mathrm{IV}$ & Surgery & $\begin{array}{c}\text { Median } 28.7 \\
(0.6-37.0)\end{array}$ & 224 & $1.04(82 / 142)$ & Multivariate & os & English & 7 \\
\hline $\begin{array}{l}\text { Ma XL } \\
(2016)^{b} \\
{[37]}\end{array}$ & Shanghai & $2012 \sim 2013$ & HCC & I $\sim$ IV & Surgery & $\begin{array}{c}\text { Median 20.3 } \\
(1.0-30.0)\end{array}$ & 219 & $1.04(55 / 164)$ & Multivariate & os & English & 7 \\
\hline $\begin{array}{l}\text { Shi H } \\
\text { (2016) [38] }\end{array}$ & Shanghai & $2009 \sim 2014$ & NSCLC & $\mathrm{IIB} \sim \mathrm{IV}$ & Mixed & $\begin{array}{l}\text { Up to Aug } \\
2015\end{array}$ & 117 & $1.2(67 / 50)$ & Multivariate & os & Chinese & 7 \\
\hline $\begin{array}{l}\text { Jiang R } \\
\text { (2014) [39] }\end{array}$ & Guangdong & $2003 \sim 2009$ & NPC & IV & Chemotherapy & $\begin{array}{l}\text { Up to Dec } \\
2013\end{array}$ & 807 & $\begin{array}{c}1.065 \\
(549 / 258)\end{array}$ & Multivariate & os & English & 7 \\
\hline
\end{tabular}

\section{Association between apoA1 and prognosis}

The main results of this meta-analysis on the association between apoA1 and prognosis are listed in Table 2. We applied a fixed-effects model to pool the HRs because of the absence of significant heterogeneity $\left(I^{2}=19.3 \%, P_{\mathrm{h}}=0.244\right)$. The combined analysis of 14 cohorts in 13 studies showed that increased apoA1 level was significantly associated with improved OS (pooled HR $=0.608,95 \% \mathrm{CI}=0.557-0.665, P<0.001$, Fig. 2 ). When subgroup analysis was conducted based on the cancer types, the overall results revealed that elevated serum apoA1 level significantly resulted in longer OS in patients with digestive system malignancies (i. e., GC, CRC, ESCC, and HCC; pooled HR $=0.633,95 \% \mathrm{CI}=0.550-0.727, P<0.001$, fixed effects), urinary system cancers (i. e., bladder cancer, UTUC, and RCC; pooled HR $=0.471,95 \%$ CI $=0.352-0.630, P<0.001$, fixed effects), NPC (pooled HR $=0.642,95 \% \mathrm{CI}=0.538-0.766$, $P<0.001$, fixed effects), and NSCLC (pooled HR $=0.526,95 \% \mathrm{CI}=0.329-0.841, P=0.007$, random effects), but this finding was not observed in patients with breast cancer (pooled $\mathrm{HR}=0.573,95 \% \mathrm{CI}=0.266-1.246, P=0.155$, random effects). In the subgroup analyses of clinical stage, higher apoA1 level was still obviously related to better OS for stage I - IV (pooled HR $=0.596,95 \% \mathrm{CI}=0.521-0.680, P<0.001$, fixed effects), and advanced stage (i. e., stage IV and IIIB - IV; pooled $\mathrm{HR}=0.626,95 \% \mathrm{CI}=0.536-0.731, P<0.001$, fixed effects), but not for early stage (i. e., stage I - III and Tis $-\mathrm{T} 1$, pooled HR $=0.539,95 \% \mathrm{CI}=0.246-1.181$, $P=0.123$, random effects). Moreover, significant association between apoA1 level and OS was found in patients who underwent surgery (pooled $\mathrm{HR}=0.596,95 \% \mathrm{CI}=0.53-0.688, P$ $<0.001$, fixed effects), chemotherapy (pooled $\mathrm{HR}=0.626,95 \% \mathrm{CI}=0.536-0.731, P<0.001$, fixed effects), radiotherapy (pooled $\mathrm{HR}=0.646,95 \% \mathrm{CI}=0.474-0.880, P=0.006$, random effects), or mixed treatment (pooled HR $=0.526,95 \% \mathrm{CI}=0.329-0.841, P=0.007$, random effects). Furthermore, the results did not markedly change when the subgroup analyses were conducted based on the study region, cutoff value, sample size, and analysis method.

Four studies comprising 4045 patients reported the primary endpoint of DFS, and the overall outcome indicated that the upregulation of serum apoA1 was associated with an extended DFS (pooled HR $=0.714,95 \% \mathrm{CI}=0.603-0.845, P<0.001$, fixed effects, Fig. 3 ), and significant heterogeneity did not exist $\left(I^{2}=32.0 \%, P_{\mathrm{h}}=0.221\right)$. 


\section{Cellular Physiology Cell Physiol Biochem 2018;51:575-588 and Biochemistry Published \begin{tabular}{l|l} 
DOI: 10.1159/000495277 & (c)18 The Author(s). Published by S. Karger AG, Basel \\
www.karger.com/cpb
\end{tabular} \\ Wu et al.: Prognostic Role of Serum Apolipoprotein A1 in Solid Tumors}

Table 2. Pooled and subgroup analysis of main results for the meta-analysis. Digestive system malignancies included gastric cancer, colorectal cancer, esophageal squamous cell cancer, and hepatocellular carcinoma. Urinary system cancers included bladder cancer, upper tract urothelial carcinoma, and renal cell cancer. Early stage included StageI III, and Stage Tis T1. Advanced stage included Stage IV and Stage IIIB IV.. Abbreviations: $\mathrm{P}_{\mathrm{z}}$ denotes $\mathrm{P}$ value for statistical significance based on $\mathrm{Z}$ test; $\mathrm{P}_{\mathrm{h}}$ denotes $\mathrm{P}$ value for heterogeneity based on $\mathrm{Q}$ test; $\mathrm{P}_{\mathrm{m}}$ denotes $\mathrm{P}$ value for statistical outcome based on multivariate metaregression analysis. HR, hazard ratio; CI, confidence interval; NPC, nasopharyngeal carcinoma; NSCLC non small cell lung cancer

\begin{tabular}{|c|c|c|c|c|c|c|c|c|}
\hline \multirow{2}{*}{ Categories } & \multirow{2}{*}{ Cohort (case) } & \multirow{2}{*}{ Model } & \multirow{2}{*}{$\operatorname{HR}(95 \% \mathrm{CI})$} & \multirow{2}{*}{$\mathrm{Z}$} & \multirow{2}{*}{$\mathrm{P}_{\mathrm{z}}$} & \multicolumn{2}{|c|}{ Heterogeneity } & \multirow{2}{*}{$\mathrm{Pm}_{\mathrm{m}}$} \\
\hline & & & & & & $\mathrm{I}^{2}$ & $\mathrm{P}_{\mathrm{h}}$ & \\
\hline Overall survival (OS) & $14(8052)$ & Fixed & $0.608(0.557 \sim 0.665)$ & 10.97 & $<0.001$ & $19.3 \%$ & 0.244 & \\
\hline Study region & & & & & & & & 0.782 \\
\hline Guangdong & $10(7022)$ & Fixed & $0.626(0.565 \sim 0.694)$ & 8.94 & $<0.001$ & $5.9 \%$ & 0.387 & \\
\hline Others & $4(1030)$ & Fixed & $0.558(0.468 \sim 0.666)$ & 6.45 & $<0.001$ & $43.6 \%$ & 0.150 & \\
\hline Cancer type & & & & & & & & 0.980 \\
\hline Digestive system & $5(2362)$ & Fixed & $0.633(0.550 \sim 0.727)$ & 6.46 & $<0.001$ & $0.0 \%$ & 0.435 & \\
\hline Urinary system & $3(1355)$ & Fixed & $0.471(0.352 \sim 0.630)$ & 5.08 & $<0.001$ & $18.2 \%$ & 0.294 & \\
\hline NPC & $2(2734)$ & Fixed & $0.642(0.538 \sim 0.766)$ & 4.91 & $<0.001$ & $0.0 \%$ & 0.961 & \\
\hline Breast cancer & $2(1343)$ & Random & $0.573(0.266 \sim 1.234)$ & 1.42 & 0.155 & $63.8 \%$ & 0.096 & \\
\hline NSCLC & $2(258)$ & Random & $0.526(0.329 \sim 0.841)$ & 2.69 & 0.007 & $70.1 \%$ & 0.068 & \\
\hline Clinical stage & & & & & & & & 0.743 \\
\hline StageI $\sim$ IV & $9(5106)$ & Fixed & $0.596(0.521 \sim 0.680)$ & 7.63 & $<0.001$ & $23.0 \%$ & 0.239 & \\
\hline Early stage & $2(1514)$ & Random & $0.539(0.246 \sim 1.181)$ & 1.54 & 0.123 & $78.2 \%$ & 0.032 & \\
\hline Advanced stage & $3(1432)$ & Fixed & $0.631(0.556 \sim 0.716)$ & 7.16 & $<0.001$ & $0.0 \%$ & 0.946 & \\
\hline Primary treatment & & & & & & & & 0.955 \\
\hline Surgery & $9(4552)$ & Fixed & $0.596(0.517 \sim 0.688)$ & 7.10 & $<0.001$ & $34.6 \%$ & 0.141 & \\
\hline Chemotherapy & $2(1315)$ & Fixed & $0.626(0.536 \sim 0.731)$ & 5.90 & $<0.001$ & $0.0 \%$ & 0.771 & \\
\hline Radiotherapy & 1 (1927) & Random & $0.646(0.474 \sim 0.880)$ & 2.77 & 0.006 & - & - & \\
\hline Mixed & $2(258)$ & Random & $0.526(0.329 \sim 0.841)$ & 2.69 & 0.007 & $70.1 \%$ & 0.068 & \\
\hline Cut-off value & & & & & & & & 0.589 \\
\hline$\geq 1.1 \mathrm{~g} / \mathrm{L}$ & 9 (5917) & Fixed & $0.619(0.558 \sim 0.688)$ & 8.96 & $<0.001$ & $36.1 \%$ & 0.130 & \\
\hline$<1.1 \mathrm{~g} / \mathrm{L}$ & $5(2135)$ & Fixed & $0.581(0.492 \sim 0.687)$ & 6.35 & $<0.001$ & $0.0 \%$ & 0.526 & \\
\hline Analysis method & & & & & & & & 0.834 \\
\hline Univariate & $3(2386)$ & Random & $0.621(0.420 \sim 0.917)$ & 2.39 & 0.017 & $64.4 \%$ & 0.060 & \\
\hline Multivariate & $11(5666)$ & Fixed & $0.595(0.538 \sim 0.657)$ & 10.16 & $<0.001$ & $0.0 \%$ & 0.480 & \\
\hline Sample size & & & & & & & & 0.230 \\
\hline$\geq 500$ & $6(6273)$ & Fixed & $0.651(0.583 \sim 0.727)$ & 7.60 & $<0.001$ & $0.0 \%$ & 0.829 & \\
\hline$<500$ & $8(1779)$ & Fixed & $0.538(0.464 \sim 0.624)$ & 8.16 & $<0.001$ & $29.3 \%$ & 0.194 & \\
\hline Disease free survival (DFS) & $4(4045)$ & Fixed & $0.714(0.603 \sim 0.845)$ & 3.92 & $<0.001$ & $32.0 \%$ & 0.221 & \\
\hline
\end{tabular}

Association between apoA1 and clinicopathological parameters

The correlations of apoA1 with clinicopathological features of solid tumors are presented in Table 3. Four cohorts with 1011 patients reported the relationship between serum apoA1 and tumor size, and the pooled outcome showed that low apoA1 level was related to larger tumor size (pooled OR $=0.640,95 \%$ CI: $0.475-0.863, P=0.003$, fixed effects). The correlation of serum apoA1 and tumor differentiation was reported in 5 studies with 1563 participants, and the conjoined result declared that decreased apoA1 level was positively correlated with poor histological grade (pooled OR $=0.724,95 \%$ CI: 0.565-0.929, $P=0.011$, fixed effects). Four studies enrolling 2425 cases exhibited the connection of apoA1 and tumor stage, and a significant association was found between low apoA1 level and advanced tumor stage (pooled OR $=0.493,95 \% \mathrm{CI}: 0.384-0.633, P<0.001$, fixed effects). However, serum apoA1 was not associated with age (pooled OR $=1.107,95 \%$ CI: $0.951-1.289, P=0.191$, fixed effects), $T$ stage (pooled OR $=0.746,95 \%$ CI: 0.429-1.297, $P=0.299$, random effects), lymph node metastasis (pooled OR $=0.785,95 \%$ CI: 0.485-1.270, $P=0.323$, random effects), and distant metastasis (pooled OR $=0.491,95 \% \mathrm{CI}$ : 0.179-1.346, $P=0.167$, random effects).

\section{Sensitivity analysis and meta-regression analysis}

The overall HR estimates for OS (Fig. 4A) or DFS (Fig. 4B) did not significantly change after the sequential omission of each single cohort. This phenomenon indicated that no individual cohort influenced the pooled results. 


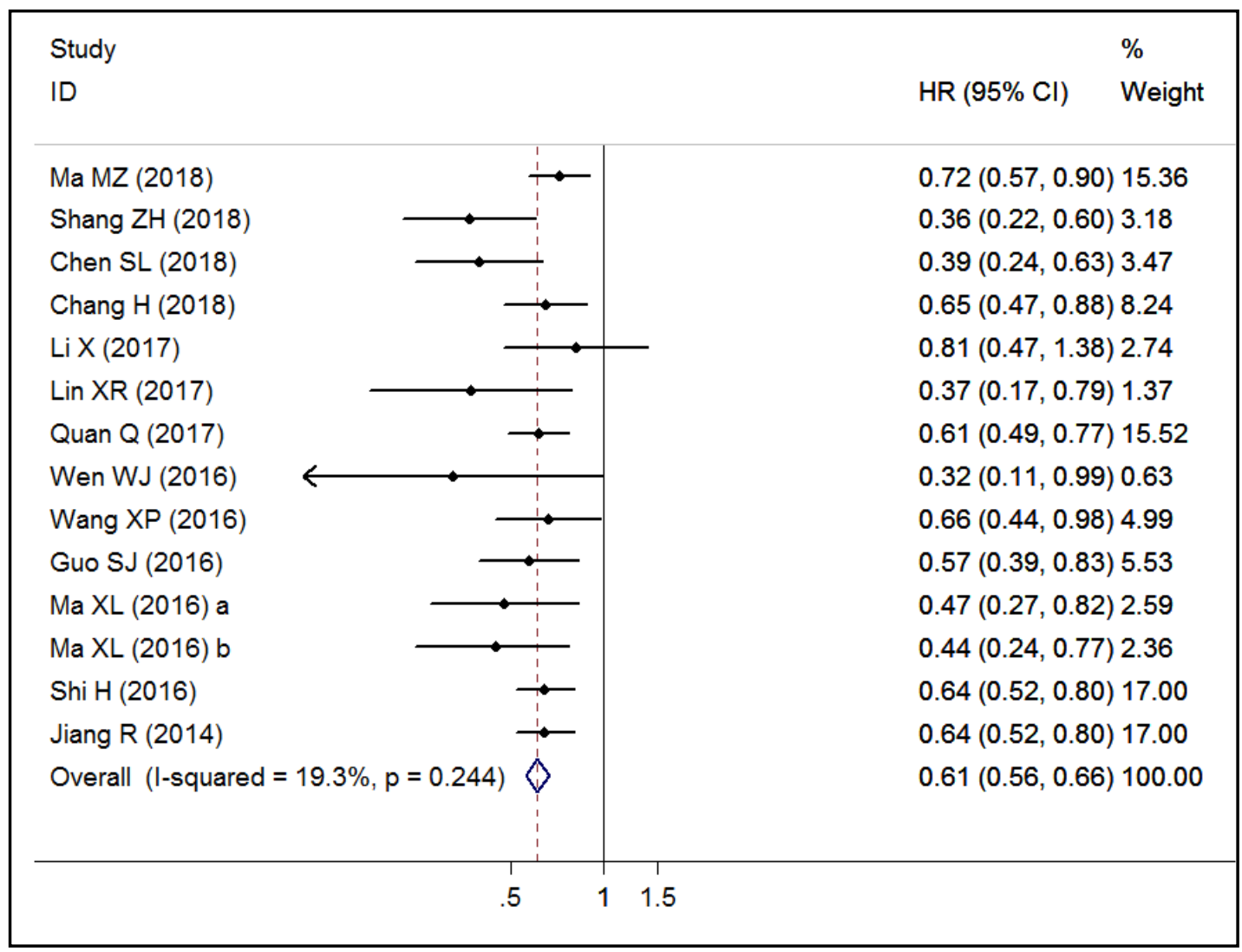

Fig. 2. Forest plots of the association between serum apolipoprotein $A 1$ and overall survival. Abbreviation: HR, hazard ratio; CI, confidence interval.

\begin{tabular}{|c|c|c|c|}
\hline Study & & & $\%$ \\
\hline ID & & $\mathrm{HR}(95 \% \mathrm{Cl})$ & Weight \\
\hline Chang H (2018) & & $0.77(0.63,0.95)$ & 63.74 \\
\hline Li X (2017) & & $0.77(0.48,1.30)$ & 11.36 \\
\hline Lin XR (2017) & & $0.38(0.20,0.75)$ & 6.70 \\
\hline Guo SJ (2016) & & $0.65(0.44,0.97)$ & 18.20 \\
\hline Overall $(I-$ squared $=32.0 \%, p=0.221)$ & & $0.71(0.60,0.84)$ & 100.00 \\
\hline .5 & 1.5 & & \\
\hline
\end{tabular}

Fig. 3. Forest plots of the association between serum apolipoprotein $\mathrm{A} 1$ and disease free survival. Abbreviation: HR, hazard ratio; $\mathrm{CI}$, confidence interval. 
Table 3. Meta-analysis of apolipoprotein A1 levels and clinicopathological parameters in solid tumors. Notes: All pooled HRs were calculated from fixed-effect model except for cells marked with (random ${ }^{R}$ ). Abbreviations: $P_{h}$ denotes $P$ value for heterogeneity based on $Q$ test; $P$ denotes $P$ value for statistical significance based on $\mathrm{Z}$ test

\begin{tabular}{lcccccc}
\hline Categories & $\begin{array}{c}\text { Studies (no. } \\
\text { of patients) }\end{array}$ & OR (95\% CI) & $\mathrm{I}^{2}(\%)$ & $\mathrm{Ph}$ & $\mathrm{Z}$ & $\mathrm{P}$ \\
\hline Age & $7(3541)$ & $1.107(0.951 \sim 1.289)$ & $0.0 \%$ & 0.587 & 1.31 & 0.191 \\
Tumor size & $5(1128)$ & $0.640(0.475 \sim 0.863)$ & $0.0 \%$ & 0.588 & 2.93 & 0.003 \\
Differentiation & $5(1563)$ & $0.724(0.565 \sim 0.929)$ & $0.0 \%$ & 0.413 & 2.54 & 0.011 \\
T stage & $4(2888)$ & $0.746(0.429 \sim 1.297)^{\mathrm{R}}$ & 85.3 & $<0.001$ & 1.04 & 0.299 \\
Tumor stage & $4(2425)$ & $0.493(0.384 \sim 0.633)$ & $0.0 \%$ & 0.587 & 5.56 & $<0.001$ \\
Lymph node metastasis & $4(2198)$ & $0.785(0.485 \sim 1.270)^{\mathrm{R}}$ & $57.1 \%$ & 0.072 & 0.99 & 0.323 \\
Distant metastasis & $2(1593)$ & $0.491(0.179 \sim 1.346)^{\mathrm{R}}$ & $85.0 \%$ & 0.010 & 1.38 & 0.167 \\
\hline
\end{tabular}

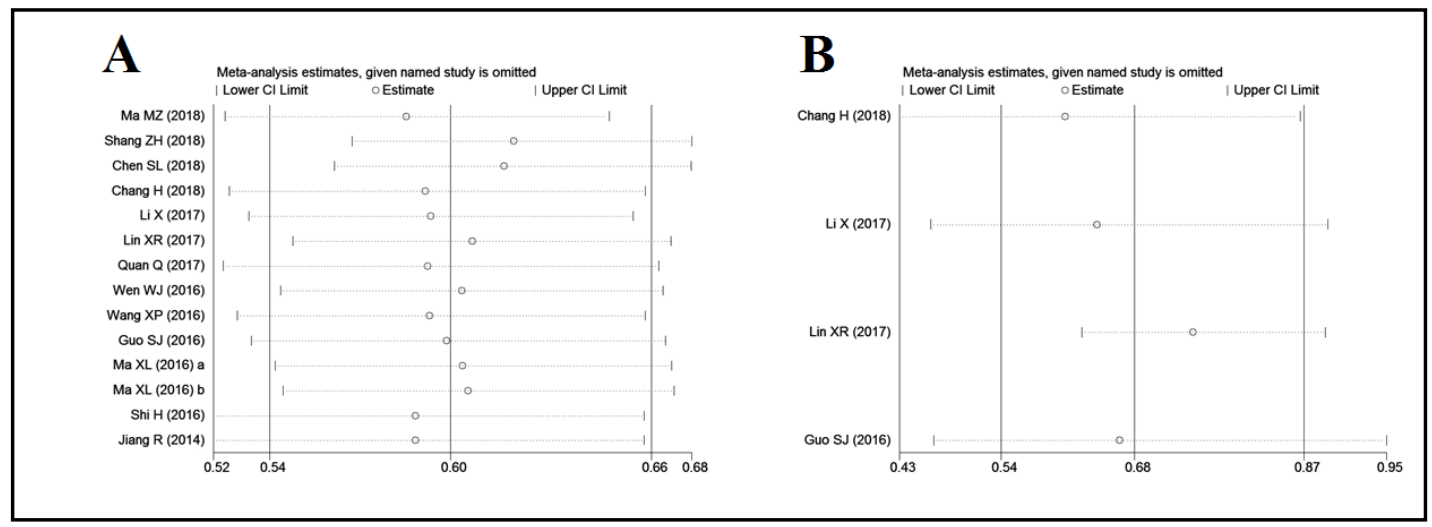

Fig. 4. Sensitivity analysis of this meta-analysis. (A) Sensitivity analysis for overall survival. (B) Sensitivity analysis for disease free survival.

We performed meta-regression analysis to investigate the potential source of heterogeneity among studies for OS. The results listed in Table 2 demonstrated that the study region $(P=0.782)$, cancer type $(P=0.980)$, clinical stage $(P=0.743)$, primary treatment $(P$ $=0.955)$, cutoff value $(P=0.589)$, analysis method $(P=0.834)$ and sample size $(P=0.230)$ did not contribute to the source of heterogeneity for OS. Moreover, we did not conduct meta-regression analysis concerning the outcome of DFS due to the insufficient number of literatures.

\section{Publication bias}

Both Begg's and Egger' tests suggested a potential publication bias with regard to the pooled outcome of OS (Begg's test, $P=0.021$; Egger' test, $P=0.005$ ), and the funnel plot also showed a certain degree of apparent asymmetry (Fig. 5A). However, the trim and fill analysis (no new studies added) showed no indication of publication bias. This phenomenon suggested that the results were robust and reliable. Moreover, Begg's test $(P=0.308)$, Egger' test $(P=0.235)$ and trim and fill method (no new studies added) showed no significant publication bias concerning the overall outcome of DFS (Fig. 5B). 


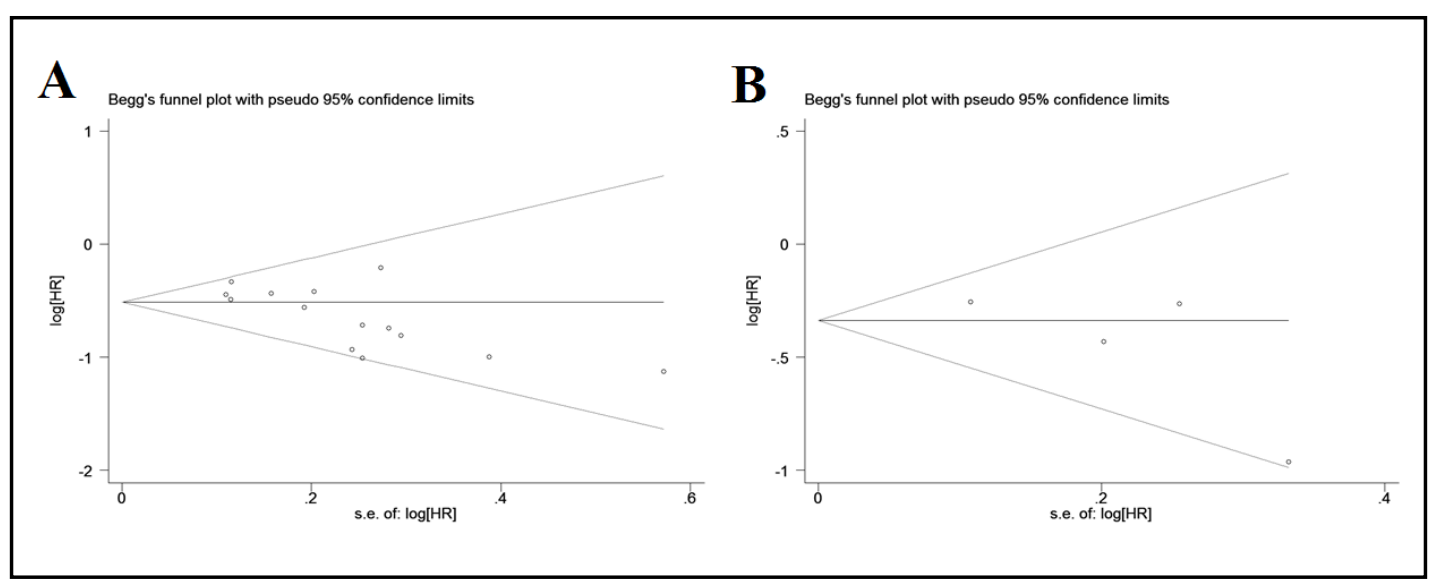

Fig. 5. Funnel plots assessing the potential publication bias of the included studies. (A) Funnel plot of publication bias for overall survival. (B) Funnel plot of publication bias for disease free survival.

\section{Discussion}

ApoA1 is a vital mediator of plasma cholesterol transport and cellular cholesterol homeostasis. This molecule is primarily synthesized by the liver and intestine, and exists transiently in lipid-poor form. Serum apoA1 is generally included as part of the routine lipid test panel at admission and before treatment in Chinese hospitals. Thus, this biomarker is a convenient and reproducible laboratory parameter. In addition to simply serving as a shuttle of cholesterol cargo during RCT, an expanded range of physiological functions of apoA1 have been described, including anti-inflammatory, anti-apoptotic, anti-parasitic, anti-bacterial, anti-viral, and anti-oxidant activities [40]. Chronic inflammation, infection, oxidative stress, and lipids have been related to tumorigenesis [41-43]. Given the beneficial role of apoA1 against atherogenic process and cardiovascular disease, apoA1 may also be protective against cancer. Numerous studies have associated the serum levels of apoA1 with the risk of developing a broad spectrum of cancers [44]. However, the prognostic value of serum apoA1 in solid tumors remained ambiguous. Thus, we performed this first meta-analysis to investigate the prognostic significance of serum apoA1 based on a data synthesis of 13 studies involving 8052 Chinese patients with various cancers. High serum apoA1 level predicted an improved OS and DFS in solid tumors, which suggested that apoA1 might serve as a positive prognostic biomarker for solid tumors. Although publication bias and sensitivity analysis have confirmed the robustness and reliability of the meta-analysis outcomes, different baseline characteristics of individual studies might lead to inter-study heterogeneity. We performed meta-regression analysis using the factors of study region, cancer type, clinical stage, primary treatment, cut-off value, analysis method, and sample size. However, none of these factors could complain the heterogeneity of OS.

Despite the robustness of the pooled results, the findings of this research should be interpreted with caution. When subgroup analysis of OS was conducted based on the cancer type, no statistical significance was found in the pooled results from patients with breast cancer, which indicated that the function of apoA1 might be tissue specific. One of the two studies that reported the association between serum apoA1 and OS in breast cancer collected 299 patients with stage I - IV and exhibited an obvious correlation [32]. The other study employed 1044 cases with stage I - III and obtained a nonsignificant relationship [31]. Thus, the inconsistencies between these studies were possibly due to the bias of sample selection and sample size. Moreover, numerous reports have focused on the role of apoA1 in breast cancer, but extremely diverse conclusions were drawn. A prospective study based on 7557 subjects [45] declared that the serum level of apoA1 was inversely associated with breast cancer risk, contrary to a recent publication by Martin [46]. Furthermore, a study has indicated that breast cancer cells with elevated apoA1 level were more sensitive 


\section{Cellular Physiology Cell Physiol Biochem 2018;51:575-588

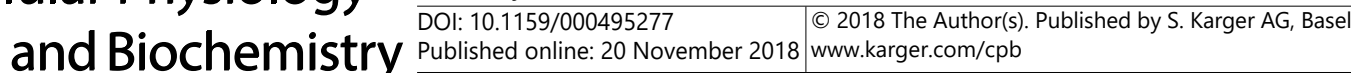 \\ Wu et al.: Prognostic Role of Serum Apolipoprotein A1 in Solid Tumors}

to chemotherapy than cells with lower apoA1 level [47]. By contrast, Hodgkinson [48] demonstrated that high apoA1 level contributed to chemotherapy resistance in breast cancer tissue with positive estrogen receptor (ER) expression. These conflicting results might be attribute to the difference in study populations and suggested that the impact of apoA1 on breast cancer might be related to ER expression. Therefore, the role of apoA1 in breast cancer remains controversial. Similarly, when the tumor stage was classified, no statistical significance was found in cancer patients with early stage (stage I - III and stage Tis - T1). Given that only two studies with 1514 cases were enrolled based on this subgroup, more well-designed studies are warranted to explore the real effect.

To verify the prognostic role of apoA1 on malignant tumors, we further analyzed the correlation between apoA1 level and clinicopathological features that may affect survival outcomes. The synthesized data suggested that decreased level of serum apoA1 was significantly correlated with some phenotypes of tumor progression, such as larger tumor size, poor histological grade, and advanced clinical stage. All these aggressive tumor behaviors have been documented to be independent factors related to tumor progression and compromise survival outcome $[49,50]$. Herein, apoA1 played an important role in the inhibition of tumor progression through different pathways. Thus, this molecule contributes to better survival in malignant patients. Therefore, cancer patients with large tumor size, poor histological grade, or advanced clinical stage may primarily benefit from serum apoA1 evaluation to make clinical decisions.

The exact mechanisms of apoA 1 anti-tumor activity remain unclear, but are undoubtly multifactorial. First, apoA1 has been proved to negatively alter tumor-permissive features of tumor microenvironment through multiple pathways. These pathways include but are not limited to the following: suppressing the increase in myeloid-derived suppressor cells to decrease tumor volume [51]; reducing the matrix metalloproteinase-9 protein level and activity to inhibit tumor metastasis [52]; increasing the recruitments of tumor cell killing macrophages and CD8 T cells [53]; reducing the level of surviving, a anti-apoptotic protein, responsible for tumor regression [54]. Second, apoA1 and apoA1 mimetic peptides can bind pro-inflammatory or pro-angiogenic phospholipids, such as lysophosphatidic acid. Moreover, these peptides can subsequently clear the phospholipids from the blood, thereby controlling cell proliferation in tumor growth [55]. Third, apoA1 affects the innate and adaptive arms of the immune system to suppress tumor-related angiogenesis, and inversely influences the migration of tumor cells. ApoA1 induces the conversion of tumor-associated macrophages from a pro-tumor M2 phenotype to an anti-tumor M1 phenotype. This process aids in cancer rejection [56]. Moreover, apoA1 also inhibits inflammation which has been inferred to participate in tumor metastasis [57].

Some potential limitations should be clarified in this meta-analysis. First, several individual HRs were obtained from univariate analysis, which have not adjusted the confounding factors and might overestimate the effect size [58]. Second, due to no acknowledged threshold, the cut-off values for defining "high" level of apoA1 were extremely different among the included studies, which might affect the availability of serum apoA1 as a prognostic biomarker in malignances. Third, the fact that studies with positive results tended to be published than works with null or negative outcomes, might lead to reporting bias and potentially exaggerate the overall outcomes. Fourth, the present meta-analysis concentrated on the Chinese population, and the results may not be able to apply to other populations. Fifth, our meta-analysis focused on pretreatment level of serum apoA1, and the clinical significance of posttreatment level, which reflects the dynamic variance of apoA1 level during tumor progression, is still unclear. Finally, the present meta-analysis only provided evidence for correlation study, which could not be simply interpreted as causal relationship. 


\section{Cellular Physiology Cell Physiol Biochem 2018;51:575-588 \begin{tabular}{ll|l} 
and Biochemistry Published online: 20 November 2018 & $\begin{array}{l}\text { (c) } 2018 \text { The Author(s). Published by S. Karger AG, Basel } \\
\text { www.karger.com/cpb }\end{array}$ \\
\hline
\end{tabular}}

Wu et al.: Prognostic Role of Serum Apolipoprotein A1 in Solid Tumors

\section{Conclusion}

In conclusion, this meta-analysis of currently available evidences supports the notion that elevated level of pretreatment serum apoA1 is significantly associated with improved prognosis in Chinese patients with solid tumors. Serum apoA1 could serve as a reliable, convenient, and low-cost biomarker for predicting the prognosis and tumor progression of solid tumors in Chinese population because of its availability as a blood routine test in daily clinical practice. In future, more experiments and clinical trials with well designs and large samples are required to verify and strengthen the prognostic significance of apoA1 in malignancies.

\section{Acknowledgements}

This study was supported by National Natural Science Foundation of China (No. 81702270), and Guangdong Province Science and Technology Development of Special Funding Support (No. 2016A020215151).

\section{Disclosure Statement}

The authors declare no conflicts of interest.

\section{References}

1 Hanahan D, Weinberg RA: Hallmarks of Cancer: The Next Generation. Cell 2011;144:646-674.

-2 Danhier P, Banski P, Payen VL, Grasso D, Ippolito L, Sonveaux P, Porporato PE: Cancer metabolism in space and time: Beyond the Warburg effect. Biochim Biophys Acta 2017;1858:556-572.

3 Poff A, Koutnik AP, Egan KM, Sahebjam S, D’Agostino D, KuMAR NB: Targeting the Warburg effect for cancer treatment: Ketogenic diets for management of glioma. Semin Cancer Biol DOI: 10.1016/j. semcancer.2017.12.011.

4 Corbet C, Feron 0: Emerging roles of lipid metabolism in cancer progression. Curr Opin Clin Nutr Metab Care 2017;20:254-260.

5 Kim SY: Cancer energy metabolism: shutting power off cancer factory. Biomol Ther (Seoul) 2018;26:39-44.

6 Goldstein JL, Brown MS: A century of cholesterol and coronaries: from plaques to genes to statins. Cell 2015;161:161-172.

7 Schwartz MW, Seeley RJ, Tschop MH, Woods SC, Morton GJ, Myers MG, D’Alessio D: Cooperation between brain and islet in glucose homeostasis and diabetes. Nature 2013;503:59-66.

8 Rosen ED, Spiegelman BM: What we talk about when we talk about fat. Cell 2014;156:20-44.

-9 Perry RJ, Samuel VT, Petersen KF, Shulman GI: The role of hepatic lipids in hepatic insulin resistance and type 2 diabetes. Nature 2014;510:84-91.

10 Long J, Zhang CJ, Zhu N, Du K, Yin YF, Tan X, Liao DF, Qin L: Lipid metabolism and carcinogenesis, cancer development. Am J Cancer Res 2018;8:778-791.

11 Liu Q, Luo Q, Halim A, Song G: Targeting lipid metabolism of cancer cells: A promising therapeutic strategy for cancer. Cancer Lett 2017;401:39-45.

12 Gordon EM, Figueroa DM, Barochia AV, Yao X, Levine SJ: High-density lipoproteins and apolipoprotein A-I: potential new players in the prevention and treatment of lung cancer. Front Pharmacol 2016;7:323.

13 Cruz PM, Mo H, McConathy WJ, Sabnis N, Lacko AG: The role of cholesterol metabolism and cholesterol transport in carcinogenesis: a review of scientific findings, relevant to future cancer therapeutics. Front Pharmacol 2013;4:119.

14 Cavigiolio G, Geier EG, Shao B, Heinecke JW, Oda MN: Exchange of apolipoprotein A-I between lipid-associated and lipid-free states: a potential target for oxidative generation of dysfunctional high density lipoproteins. J Biol Chem 2010;285:18847-18857. 


\section{Cellular Physiology Cell Physiol Biochem 2018;51:575-588 and Biochemistry DOl: 10.1159/000495277 2010 (O) 2018 The Author(s). Published by S. Karger AG, Basel

15 Curtiss LK, Valenta DT, Hime NJ, Rye KA: What is so special about apolipoprotein AI in reverse cholesterol transport? Arterioscler Thromb Vasc Biol 2006;26:12-19.

16 Nolen BM, Lokshin AE: Biomarker testing for ovarian cancer: clinical utility of multiplex assays. Mol Diagn Ther 2013;17:139-146.

17 Chandler PD, Song Y, Lin J, Zhang S, Sesso HD, Mora S, Giovannucci EL, Rexrode KE, Moorthy MV, Li C, Ridker PM, Lee IM, Manson JE, Buring JE, Wang L: Lipid biomarkers and long-term risk of cancer in the Women's Health Study. Am J Clin Nutr 2016;103:1397-1407.

18 van Duijnhoven FJ, Bueno-De-Mesquita HB, Calligaro M, Jenab M, Pischon T, Jansen EH, Frohlich J, Ayyobi A, Overvad K, Toft-Petersen AP, Tjønneland A, Hansen L, Boutron-Ruault MC, Clavel-Chapelon F, Cottet V, Palli D, Tagliabue G, Panico S, Tumino R, Vineis P, Kaaks R et al.: Blood lipid and lipoprotein concentrations and colorectal cancer risk in the European Prospective Investigation into Cancer and Nutrition. Gut 2011;60:1094-1102.

19 Su F, Kozak KR, Imaizumi S, Gao F, Amneus MW, Grijalva V, Ng C, Wagner A, Hough G, Farias-Eisner G, Anantharamaiah GM, Van Lenten BJ, Navab M, Fogelman AM, Reddy ST, Farias-Eisner R: Apolipoprotein A-I (apoA-I) and apoA-I mimetic peptides inhibit tumor development in a mouse model of ovarian cancer. Proc Natl Acad Sci U S A 2010;107:19997-20002.

20 Zamanian-Daryoush M, Lindner D, Tallant TC, Wang Z, Buffa J, Klipfell E, Parker Y, Hatala D, ParsonsWingerter P, Rayman P, Yusufishaq MS, Fisher EA, Smith JD, Finke J, DiDonato JA, Hazen SL: The cardioprotective protein apolipoprotein A1 promotes potent anti-tumorigenic effects. J Biol Chem 2013;288:21237-21252.

-21 Clarke CH, Yip C, Badgwell D, Fung ET, Coonbes KR, Zhang Z, Lu KH, Bast RC Jr: Proteomic biomarkers apolipoprotein $\mathrm{A} 1$, truncated transthyretin and connective tissue activating protein III enhance the sensitivity of CA125 for detecting early stage epithelial ovarian cancer. Gynecol Oncol 2011;122:548-553.

-22 Nosov V, Su F, Amneus M, Birrer M, Robins T, Kotlerman J, Reddy S, Farias-Eisner R: Validation of serum biomarkers for detection of early-stage ovarian cancer. Am J Obstet Gynecol 2009;200:639.

-23 Moher D, Liberati A, Tetzlaff J, Altman DG: Preferred reporting items for systematic reviews and metaanalyses: the PRISMA statement. Int J Surg 2010;8:336-341.

$24 \mathrm{Wu}$, Hu L, Chen M, Cao W, Chen H, He T: Pyruvate kinase M2 overexpression and poor prognosis in solid tumors of digestive system: evidence from 16 cohort studies. Onco Targets Ther 2016;9:4277-4288.

25 Egger M, Davey SG, Schneider M, Minder C: Bias in meta-analysis detected by a simple, graphical test. BMJ 1997;315:629-634.

26 Peters JL, Sutton AJ, Jones DR, Abrams KR, Rushton L: Performance of the trim and fill method in the presence of publication bias and between-study heterogeneity. Stat Med 2007;26:4544-4562.

-27 Ma MZ, Yuan SQ, Chen YM, Zhou ZW: Preoperative apolipoprotein B/apolipoprotein A1 ratio: a novel prognostic factor for gastric cancer. Onco Targets Ther 2018;11:2169-2176.

28 Shang Z, Wang J, Wang X, Yan H, Cui B, Jia C, Wang Q, Cui X, Li J, Ou T: Preoperative serum apolipoprotein A-I levels predict long-term survival in non-muscle-invasive bladder cancer patients. Cancer Manag Res 2018;10:1177-1190.

29 Chen S, Lai Y, He Z, Li J, He X, Shen R, Ding Q Chen H, Peng S, liu W: Establishment and validation of a predictive nomogram model for non-small cell lung cancer patients with chronic hepatitis. J Transl Med 2018;16:116.

-30 Chang H, Wei JW, Chen K, Zhang S, Han F, Lu LX, Xiao WW, Gao YH: Apolipoprotein A-I is a prognosticator of nasopharyngeal carcinoma in the era of intensitymodulated radiotherapy. J Cancer 2018;9:702-710.

31 Li X, Tang H, Wang J, Xie X, Liu P, Kong Y, Ye F, Shuang Z, Xie Z, Xie X: The effect of preoperative serum triglycerides and high-density lipoprotein-cholesterol levels on the prognosis of breast cancer. Breast 2017;32:1-6.

32 Lin X, Hong S, Huang J, Chen Y, Chen Y, Wu Z: Plasma apolipoprotein A1 levels at diagnosis are independent prognostic factors in invasive ductal breast cancer. Discov Med 2017;23:247- 258.

-33 Quan Q, Huang Y, Chen Q, Qiu H, Hu Q Rong Y, Li T, Xia L, Zhang B: Impact of serum apolipoprotein A-I on prognosis and bevacizumab efficacy in patients with metastatic colorectal cancer. Transl Oncol 2017;10:288-294.

34 Wen WJ, Ye YL, Chen MK, Qin ZK: Serum apolipoprotein A-1 predicts fuperior prognosis in upper urothelial carcinoma after rapid surgery. Chin J Endourol 2016;10:48-51. 


\section{Cellular Physiology Cell Physiol Biochem 2018;51:575-588 \begin{tabular}{l|l|l|l} 
DOl: 10.1159/000495277 & 2018 The Author(s). Published by S. Karger AG, Basel
\end{tabular}

35 Wang XP, Li XH, Zhang L, Lin JH, Huang H, Kang T, Mao MJ, Chen H, Zheng X: High level of serum apolipoprotein A-I is a favorable prognostic factor for overall survival in esophageal squamous cell carcinoma. BMC Cancer 2016;16:516.

36 Guo S, He X, Chen Q Yang G, Yao K, Dong P, Ye Y, Chen D, Zhang Z, Qin Z, Liu Z, Li Z, Xue Y, Zhang M, Liu $\mathrm{R}$, Zhou F, Han H: The effect of preoperative apolipoprotein A-I on the prognosis of surgical renal cell carcinoma: a retrospective large sample study. Medicine 2016;95:e3147.

-37 Ma XL, Gao XH, Gong ZJ, Wu J, Tian L, Zhang CY, Zhou Y, Sun YF, Hu B, Qiu SJ, Zhou J, Fan J, Guo W, Yang XR: Apolipoprotein A1: a novel serum biomarker for predicting the prognosis of hepatocellular carcinoma after curative resection. Oncotarget 2016;7:70654-70668.

-38 Shi H, Sun Q Han Y, Dong Y, Bai C: Level of serum apolipoprotein A-1 in patients with advanced non-small cell lung cancer and its clinical significance. Chin Clin Oncol 2016;21:146-151.

-39 Jiang R, Yang ZH, Luo DH, Guo L, Sun R, Chen QY, Huang PY, Qiu F, Zou X, Cao KJ, Mai HQ Guo CN, Hong $\mathrm{MH}$, Chen MY: Elevated apolipoprotein A-I levels are associated with favorable prognosis in metastatic nasopharyngeal carcinoma. Med Oncol 2014;31:80.

40 Mangaraj M, Nanda R, Panda S: Apolipoprotein A-I: a molecule of diverse function. Indian J Clin Biochem 2016;31:253-259.

41 Kobayashi Y, Kashima H, Wu RC, Jung JG, Kuan JC, Gu J, Xuan J, Sokoll L, Visvanathan K, Shih IeM, Wang TL: Mevalonate pathway antagonist suppresses formation of serous tubal intraepithelial carcinoma and ovarian carcinoma in mouse models. Clin Cancer Res 2015;21:4652-4662.

42 Noguti J, Andersen ML, Cirelli C, Ribeiro DA: Oxidative stress, cancer, and sleep deprivation: is there a logical link in this association? Sleep Breath 2013;17:905-910.

43 Coussens LM, Zitvogel L, Palucka AK: Neutralizing tumor-promoting chronic inflammation: a magic bullet? Science 2013;339:286-291.

-44 Zamanian-Daryoush M, DiDonato JA: Apolipoprotein A-I and cancer. Front Pharmacol 2015;6:265.

45 His M, Zelek L, Deschasaux M, Pouchieu C, Kesse-Guyot E, Hercberg S, Galan P, Latino-Martel P, Blacher J, Touvier M: Prospective associations between serum biomarkers of lipid metabolism and overall, breast and prostate cancer risk. Eur J Epidemiol 2014;29:119-132.

46 Martin LJ, Melnichouk O, Huszti E, Connelly PW, Greenberg CV, Minkin S, Boyd NF: Serum lipids, lipoproteins, and risk of breast cancer: a nested case-control study using multiple time points. J Natl Cancer Inst 2015;107:djv032.

47 Cortesi L, Barchetti A, De Matteis E, Rossi E, Della Casa L, Marcheselli L, Tazzioli G, Lazzaretti MG, Ficarra G, Federico M, Iannone A: Identification of protein clusters predictive of response to chemotherapy in breast cancer patients. J Proteome Res 2009;8:4916-4933.

48 Hodgkinson VC, Agarwal V, ELFadl D, Fox JN, McManus PL, Mahapatra TK, Kneeshaw PJ, Drew PJ, Lind MJ, Cawkwell L: Pilot and feasibility study: comparative proteomic analysis by 2-DE MALDI TOF/TOF MS reveals 14-3-3 proteins as putative biomarkers of response to neoadjuvant chemotherapy in ER-positive breast cancer. J Proteomics 2012;75:2745-2752.

-49 Wu J, Liang C, Chen M, Su W: Association between tumor-stroma ratio and prognosis in solid tumor patients: a systematic review and meta-analysis. Oncotarget 2016;7:68954-68965.

50 Wu J, Hu L, Chen M, Cao W, Chen H, He T: Pyruvate kinase M2 overexpression and poor prognosis in solid tumors of digestive system: evidence from 16 cohort studies. Onco Targets Ther 2016;9:4277-4288.

-51 Neyen C, Pluddemann A, Mukhopadhyay S, Maniati E, Bossard M, Gordon S, Hagemann T: Macrophage scavenger receptor a promotes tumor progression in murine models of ovarian and pancreatic cancer. J Immunol 2013;190:3798-3805.

52 Gao F1, Vasquez SX, Su F, Roberts S, Shah N, Grijalva V, Imaizumi S, Chattopadhyay A, Ganapathy E, Meriwether D, Johnston B, Anantharamaiah GM, Navab M, Fogelman AM, Reddy ST, Farias-Eisner R: L-5F, an apolipoprotein A-I mimetic, inhibits tumor angiogenesis by suppressing VEGF/basic FGF signaling pathways. Integr Biol (Camb) 2011;3:479-489.

53 Peng M, Zhang Q, Cheng Y, Fu S, Yang H, Guo X, Zhang J, Wang L, Zhang L, Xue Z, Li Y, Da Y, Yao Z, Qiao L, Zhang R: Apolipoprotein A-I mimetic peptide 4F suppresses tumor- associated macrophages and pancreatic cancer progression. Oncotarget 2017;8:99693-99706.

-54 Feuerborn R, Becker S, Potì F, Nagel P, Brodde M, Schmidt H, Christoffersen C, Ceglarek U, Burkhardt R, Nofer JR: High density lipoprotein (HDL)-associated sphingosine 1-phosphate (S1P) inhibits macrophage apoptosis by stimulating STAT3 activity and survivin expression. Atherosclerosis 2017;257:29-37. 


\section{Cellular Physiology Cell Physiol Biochem 2018;51:575-588 and Biochemistry $\begin{gathered}\text { DOl: 10.1159/000495277 } \\ \text { Published }\end{gathered}$

Wu et al.: Prognostic Role of Serum Apolipoprotein A1 in Solid Tumors

55 Li H, Wang D, Zhang H, Kirmani K, Zhao Z, Steinmetz R, Xu Y: Lysophosphatidic acid stimulates cell migration, invasion, and colony formation as well as tumorigenesis/metastasis of mouse ovarian cancer in immunocompetent mice. Mol Cancer Ther 2009;8:1692-1701.

-56 Murphy AJ, Westerterp M, Yvan-Charvet L, Tall AR: Anti-atherogenic mechanisms of high density lipoprotein: effects on myeloid cells. Biochim Biophys Acta 2012;1821:513-521.

-57 Sirniö P, Väyrynen JP, Klintrup K, Mäkelä J, Mäkinen MJ, Karttunen TJ, Tuomisto A. Decreased serum apolipoprotein A1 levels are associated with poor survival and systemic inflammatory response in colorectal cancer. Sci Rep 2017;7:5374.

-58 Zheng X, Song X, Shao Y, Xu B, Hu W, Zhou Q, Chen L, Zhang D, Wu C, Jiang J: Prognostic role of tumorinfiltrating lymphocytes in esophagus cancer: a meta-analysis. Cell Physiol Biochem 2018;45:720-732. 